\title{
Segmental Bone Loss of the Clavicle due to Fracture-Related Infection: A Multidisciplinary Challenge
}

Femke Goormans ${ }^{1}$, Jan Vranckx², Michiel Herteleer ${ }^{1}$, Jolien Onsea ${ }^{1,5}$, Nathalie Noppe ${ }^{3}$, Melissa Depypere , Stefaan Nijs 1,5 , Willem-Jan Metsemakers ${ }^{1,5}$

1. University Hospitals Leuven, Department of Trauma Surgery, B-3000 Leuven, Belgium

2. University Hospitals Leuven, Department of Plastic and Reconstructive Surgery, B-3000 Leuven, Belgium

3. University Hospitals Leuven, Department of Radiology, B-3000 Leuven, Belgium

4. University Hospitals Leuven, Department of Laboratory Medicine, B-3000 Leuven, Belgium

5. KU Leuven - University of Leuven, Department Development and Regeneration, B-3000 Leuven, Belgium

$\triangle$ Corresponding author: Willem-Jan Metsemakers, Department of Trauma Surgery, University Hospitals Leuven, Herestraat 49, 3000, Leuven, Belgium. E-mail address: willem-jan.metsemakers@uzleuven.be

(C) Ivyspring International Publisher. This is an open access article distributed under the terms of the Creative Commons Attribution (CC BY-NC) license (https://creativecommons.org/licenses/by-nc/4.0/). See http://ivyspring.com/terms for full terms and conditions.

Received: 2018.12.26; Accepted: 2019.01.26; Published: 2019.02.16

\begin{abstract}
As the surgical treatment of clavicular fractures is increasingly utilized in orthopedics, the number of related complications rises as well. We present a case of segmental bone loss of the clavicle due to fracture-related infection. Treatment was based on a multidisciplinary team approach, which was important for a successful outcome.
\end{abstract}

Key words: fracture-related infection, bone defect, clavicle fracture, osteocutaneous free fibula flap, multidisciplinary team, MDT

\section{Introduction}

Fracture-related infection (FRI) is a severe complication that can occur after operative treatment of fractures of the clavicle. As the surgical treatment of clavicular fractures is increasingly utilized in orthopedics today, the number of complications occurring after fracture fixation will rise as well [1]. In rare cases, FRI in this region can lead to severe osteocutaneous defects, which require reconstruction in order to achieve good anatomic, cosmetic and functional restoration of the shoulder girdle.

We present a multidisciplinary team (MDT) approach for the planning and reconstruction of segmental bone loss of the clavicle due to FRI. Definitive surgical treatment consisted of an osteocutaneous free fibula transfer.

\section{Planning and surgical technique}

A 17-year-old female patient sustained a fracture of the left clavicle after a fall (Figure 1A). The initial treatment, performed at an outlying hospital, was screw osteosynthesis. This treatment failed after 6 days (Figure 1B), and a revision plate osteosynthesis was then performed (Figure 1C). The spontaneous seropurulent discharge 4 days postoperative was initially treated conservatively with antibiotics. After 2 weeks of continuous drainage, operative debridement, irrigation and implant retention was the treatment of choice.

After another 2 weeks of recuperation, and more than a month of antibiotic therapy, the patient presented to our emergency department (tertiary referral center) with a wound fistula (a confirmatory criterion for FRI [2]) and intermittent shoulder pain during mobilization. Inflammatory markers fell within the range of normal values. C-reactive protein (CRP) level was $1.5 \mathrm{mg} / \mathrm{L}$ (range of normal values: $\leq 5$ $\mathrm{mg} / \mathrm{L}$ ), with a white blood cell (WBC) count of $9.98 \mathrm{x}$ $10^{9} / \mathrm{L}$ (range of normal values: $4-10 \times 10^{9}$ ). 
An MDT meeting, consisting of trauma surgeons, microbiologists, pharmacists, radiologists, plastic and reconstructive surgeons, was convened. Thereafter the treatment plan, which will be described below, was discussed with patient and family. The first stage of treatment consisted of debridement and irrigation, combined with hardware removal. The latter was necessary due to implant loosening. Samples of soft tissue and bone were sent for bacterial analysis. The resulting cultures were positive for Pseudomonas spp. susceptible to ceftazidime, which was administered intravenously for three weeks $(3 \times 2$ grams/day). Meaning a total duration of two weeks after the bone resection. As all dead and infected bone tissue was removed, the MDT decided that it was sufficient to give two weeks of antimicrobial therapy to eradicate the residual contamination in the soft tissue.

As during surgery it became clear that the amount of periosteal stripping and bony involvement was extensive, it was decided to plan further imaging to allow a better visualization of the extent of the infection after implant removal (i.e. 3-stage approach). An extensive bone resection could namely have the result that future plate fixation would no longer be possible, especially on the lateral aspect of the clavicle. Postoperative evaluation with computed tomography (CT) indeed helped determine the extent of bony resection that would treat the infection and still allow reconstruction (Figure 2).

When the culture results were available, after approximately one week, $8 \mathrm{~cm}$ of infected necrotic bone was resected (second stage). Considering the susceptibility pattern of the Pseudomonas spp., a PMMA (polymethyl methacrylate) cement spacer containing gentamicin was implanted for local drug delivery (Figure 3). Histopathology confirmed the diagnosis of infection and described an acute purulent component with the presence of a high number of polymorphonuclear cells (PMNs). Furthermore, bone necrosis (i.e. sequestration) in combination with the presence of osteomyelitis (chronic component) could be visualized.
Considering a number of factors, including the previous long and complex infectious process, the extent of bone loss, the soft tissue status and the patient's age, the MDT decided to reconstruct the bone defect using an osteocutaneous free fibula flap. The vascularized flap was not only used to bridge the defect created by removal of the affected clavicle, but also to improve the soft tissue status (i.e. skin closure).

Although plain radiography gave an impression of length and axis, for the exact anatomical reconstruction of the clavicle, three-dimensional (3D) planning using $\mathrm{CT}$ proved to be considerably more precise [3]. Therefore, in this case we used 3D planning as a tool to measure the necessary length and axis correction, which led to a detailed descripttion of the requirements for the osteocutaneous free fibula flap.

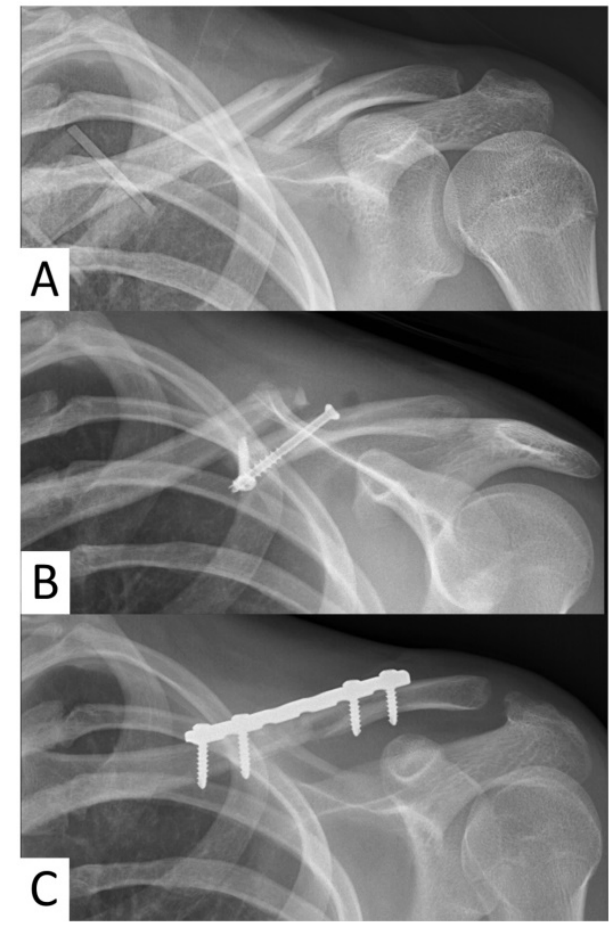

Figure 1. Plain anteroposterior radiograph of the left clavicle (A) showing the fracture that the patient sustained after a fall, (B) the failed primary (screw) osteosynthesis, and (C) the revision plate osteosynthesis that was performed after the initially failed osteosynthesis.

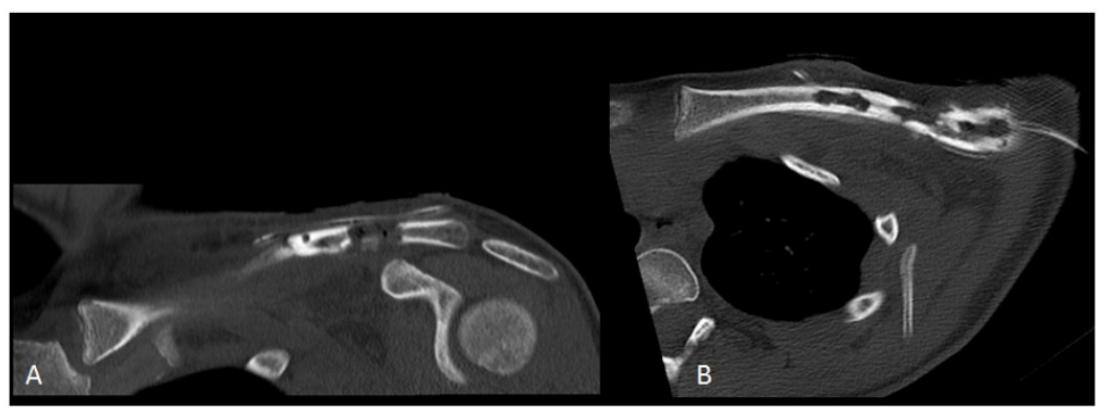

Figure 2. (A and B) Axial computed tomography (CT) scan images of the left clavicle showing the extent of the osteomyelitis after removing the plate and screw osteosynthesis. 


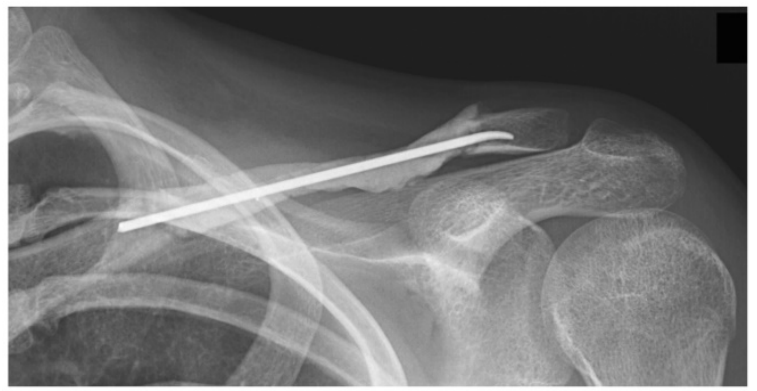

Figure 3. Plain anteroposterior radiograph of the left clavicle. After resection of the necrotic bone, a PMMA (polymethyl methacrylate) cement spacer was placed and fixated intramedullary with a titanium elastic nail (TEN).

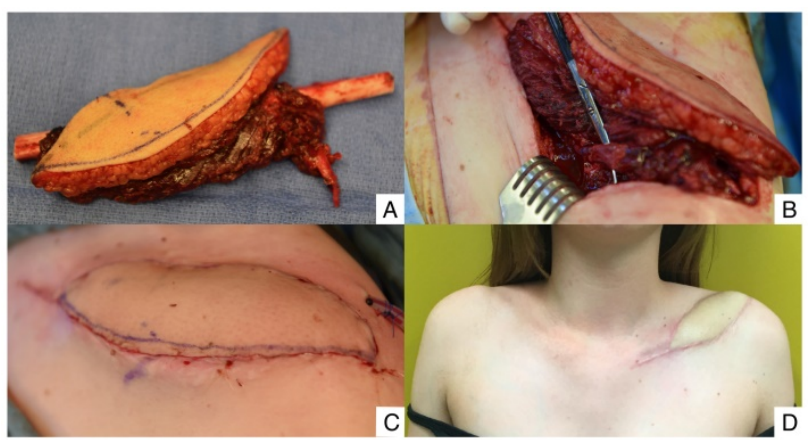

Figure 4. (A) Image showing the osteocutaneous free fibula flap. (B) Image showing the microvascular anastomosis of the fibular vascular peroneal artery on the thoracoacromial artery branch. (C) Image showing the skin island that provided for a vascularised skin cover over the revascularized fibula, which allowed for efficient monitoring of the fibula flap after microvascular anastomosis. (D) Image showing the skin island over the fibula flap that healed well, supplying soft well vascularized elastic skin cover over the restored clavicle.

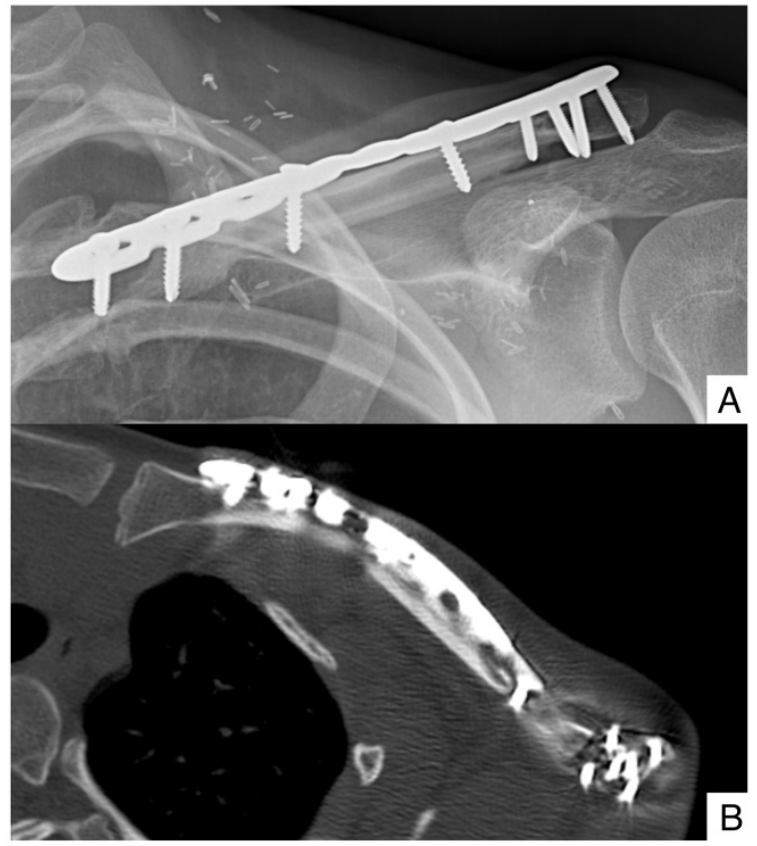

Figure 5. (A) Plain anteroposterior radiograph of the left clavicle. (B) Computed tomography (CT) scan images of the left clavicle. The images illustrate the neo-clavicle after the reconstruction of the bone defect, using an osteocutaneous free fibula flap and a superior anterior clavicle plate osteosynthesis. There is complete bony consolidation at the interface between the clavicular ends and the fibula flap both medially and laterally.
Three months after the last debridement and after an antibiotic-free interval of 6 weeks, definitive reconstruction of the clavicle was performed (third stage). In this rare case, the MDT decided to have a long antibiotic-free (holiday) interval of 6 weeks to allow planning, improve soft tissue status and of course ensure that there was no recurrence of infection.

In a final stage, the PMMA cement spacer was removed and cultures were taken. Thereafter, the osteocutaneous free fibula flap was harvested from the ipsilateral leg. To secure skin perfusion we included one osteocutaneous perforator running in the lateral septum in the distal third of the lower extremity [4]. The flap (Figure 4A) was positioned in a 'tight-fit' fashion between both clavicular ends and fixed by a commercial plate osteosynthesis (LCP Superior Anterior Clavicle Plate, DepuySynthes). End-to-end anastomosis of the fibular vascular peroneal artery and one concomitant vein was performed on a thoracoacromial artery branch and the cephalic vein, respectively (Figure 4B). The skin island provided a soft vascularized skin cover over the revascularized fibula and allowed for efficient monitoring of the fibula after microvascular anastomosis (Figure 4C).

The postoperative course was uneventful, and the patient was discharged 2 weeks after surgery. Definitive culture results didn't show growth of Pseudomonas spp. The affected shoulder was immobilized for 2 weeks postoperatively with a shoulder abduction brace, which was followed by a restriction of shoulder abduction over 90 degrees for 4 weeks. After that, the patient was allowed gradual return to full range of motion. The skin island over the fibula flap healed well, supplying a vascularized skin cover over the restored clavicle (Figure 4D).

Plain radiographs (Figure 5A) and a CT-scan (Figure 5B), obtained 18 months postoperatively, confirmed complete bony consolidation at the interface between the clavicular ends and the fibula flap. A 3D evaluation showed restoration of the anatomy of the shoulder girdle (Figures 6 and 7). Figure 8 depicts the function 2 years after surgery. Clinically, there was no shoulder pain or limited movement and the patient reported a full return to activities.

\section{Discussion}

Operative treatment of clavicular fractures lowers non-union and mal-union rates and improves functional outcome and pain scores [5]. As the number of such surgical interventions consequently rises, the number of postoperative infectious complications will rise as well [1]. Surgeons should be 
aware that FRI, especially in combination with segmental bone loss, can become a significant clinical problem. These complex revision cases demand individualized patient care and an MDT approach to improve outcome. This report describes such an approach for the planning and treatment of a complex revision case. Long-term outcome was described in detail, as this has been lacking in previous publications [6].

Depending on the extent of bone loss, the soft tissue status, and institutional preferences, several surgical and non-surgical management options may be considered for reconstruction when the infection is under control. For small bone defects, treatment using autologous bone grafts or bone substitutes has shown good results. However, for larger bone defects, more invasive treatment methods are often required [7]. Although osteocutaneous free fibula transfer was preferred in our case, previous studies also reported a good outcome with distraction osteogenesis at the level of the clavicle [8]. Furthermore, recent studies showed acceptable results in case of smaller defects up to $4 \mathrm{~cm}$ - using the induced membrane (Masquelet) technique [9]. In this case, with a defect size of $8 \mathrm{~cm}$, both techniques would not be able to create enough construct stability. For this reason, it was also decided to keep the structural integrity of the fibula without performing osteotomies to recreate the curvature of the clavicle. With help of preoperative 3D planning a tight press-fit of the fibula between both clavicular ends could be accomplished, in combination with the restoration of the correct sternoacromial distance (i.e. length), accepting some loss of the anatomic curvature (Figure 8). Stability is of paramount importance not only to achieve bone union, but also for prevention and treatment of infection. In the early phases of onset of FRI, for example, consolidation can be achieved despite the presence of an infection, as long as the construct (i.e. fracture - implant) remains stable. If stability is not granted, like in the case presented here, hardware removal (or exchange) should be performed [1]. The experimental studies by Rittmann and Perren, showed the positive influence of stability on infection in fracture care [10]. They stated that the advantage of the stabilizing effect of implants outweighs the disadvantage of a foreign body effect.

Except stability and restoring the clavicular anatomy, another reason to choose for an osteocutaneous free fibula transfer in this anatomic region is the complex blood supply [11]. Havet et al. reported that the main blood supply to the middle third of the clavicle is periosteal. If this periosteal blood supply cannot be preserved during surgical treatment in this region, vascularized bone grafting is indicated [12].

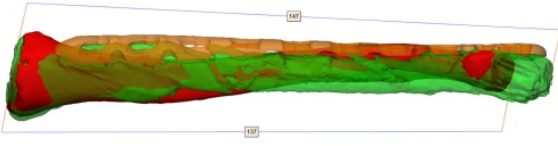

A

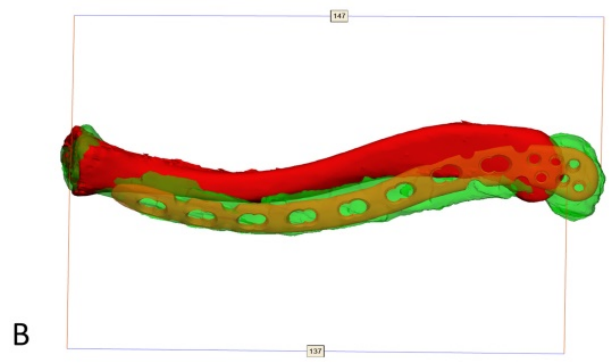

Figure 6. (A) Anteroposterior view of 3-dimensional reconstructed CT images of the clavicles. (B) Superior view of 3-dimensional reconstructed CT images of the clavicles. The 3-dimensional images illustrate the clavicle that was operatively treated with an osteocutaneous free fibula flap (green) as well as the mirrored contralateral clavicle (red). The images were segmented and aligned in Mimics innovation suite 20.0 (Materialise, Leuven, Belgium). The plate position is added for demonstrational purposes.

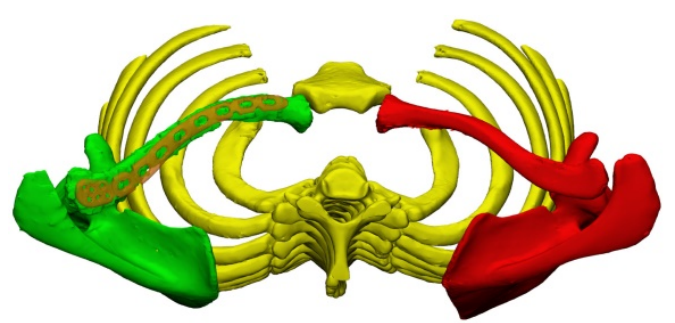

Figure 7. Thoracic inlet view of 3-dimensional reconstructed CT images of the chest wall, scapulae and clavicles. The 3-dimensional images illustrate the clavicle that was operatively treated with an osteocutaneous free fibula flap (green) as well as the contralateral clavicle (red). The scapulae have the same coloration as the adjacent clavicles. The images were segmented and aligned in Mimics innovation suite 20.0 (Materialise, Leuven, Belgium). The plate position is added for demonstrational purposes.

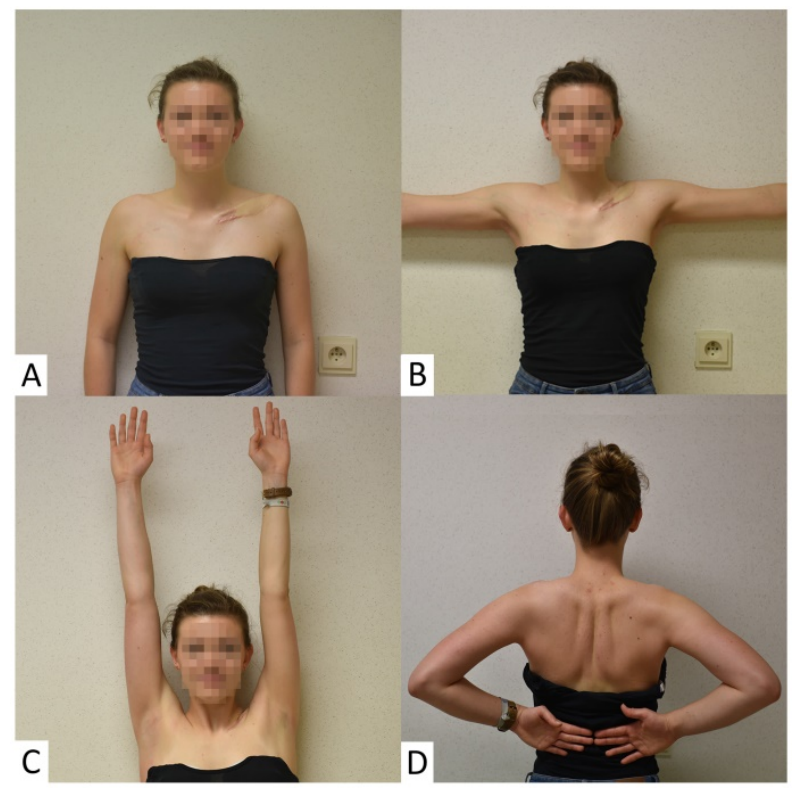

Figure 8. Shoulder function: (A) neutral position, (B) scapular abduction, (C) anteflexion and (D) internal rotation. 
Free fibula transfer has become a frequently used technique for the reconstruction of long bone defects, especially at the level of the upper extremity [7]. Because of its abundant vascularity, the osteocutaneous free fibula flap stimulates osteogenesis with new healthy and vascularized tissue in an atrophic or septic environment [6], making it an interesting treatment strategy in cases of FRI. In case of the clavicle, the thoracoacromial trunk artery and vein as well as the descending genicular vessels are constantly present and are therefore available for reliable microsurgical anastomoses [11].

In conclusion, the importance of a multidisciplinary approach for prevention and treatment of patients with FRI has recently gained attention [2, 11, 12]. This report confirms that especially planning and treatment of complex revision cases requires dedication from an MDT. Therefore, it should be strongly considered to treat these patients in centers with the availability of multiple medical specialties (e.g. trauma surgeons, infectious disease specialists, radiologists, plastic and reconstructive surgeons) experienced in the treatment of these cases.

\section{Conclusion}

FRI is one of the most challenging complications in orthopedic trauma surgery and may be exceptionally difficult to treat. It can lead to extensive bone loss and permanent disability. Our report confirms that at the level of the clavicle this sometimes necessitates complex procedures to restore anatomy and function. In these challenging revision cases an MDT approach is highly recommended to achieve a successful outcome.

\section{Competing Interests}

The authors have declared that no competing interest exists.

\section{References}

1. Metsemakers WJ, Kuehl R, Moriarty TF, Richards RG, Verhofstad M, Borens $\mathrm{O}$, et al. Infection after fracture fixation: current surgical and microbiological concepts. Injury. 2018; 49: 511-22.

2. Metsemakers WJ, Morgenstern M, McNally MA, Moriarty TF, McFadyen I, Scarborough M, et al. Fracture-related infection: a consensus on definition from an international expert group. Injury. 2018; 49: 505-10.

3. Omid R, Kidd C, Yi A, Villacis D, White E. Measurement of clavicle fracture shortening using computed tomography and chest radiography. Clin Orthop Surg. 2016; 8: 367-72.

4. Vrints I, Depreitere B, Vranckx JJ. Multilevel cervical reconstruction with no remaining hardware: the potential of a vascularised fibular strut graft. J Plast Reconstr Aesthet Surg. 2012; 65: 344-7.

5. Melean PA, Zuniga A, Marsalli M, Fritis NA, Cook ER, Zilleruelo M, et al. Surgical treatment of displaced middle-third clavicular fractures: a prospective, randomized trial in a working compensation population. J Shoulder Elbow Surg. 2015; 24: 587-92.

6. Abarca J, Valle P, Valenti P. Clavicular reconstruction with free fibula flap: a report of four cases and review of the literature. Injury. 2013; 44: 283-7.

7. Kadhim M, Holmes L, Gesheff MG, Conway JD. Treatment options for nonunion with segmental bone defects: systematic review and quantative evidence synthesis. J Orthop Trauma. 2017; 31: 111-9.
8. Sewell MD, Higgs DS, Lambert SM. Clavicle lengthening by distraction osteogenesis for congenital clavicular hypoplasia: case series and description of technique. J Pediatr Orthop. 2013; 33: 314-20.

9. Calori GM, Mazza EL, Colombo A, Mazzola S, Colombo M. Treatment of an atrophic clavicle non-union with the chamber induction technique: a case report. Injury. 2017; 48: 71-5.

10. Rittmann WW, Perren SM. Cortical bone healing after internal fixation and infection: biomechanics and biology. Berlin: Springer. 1974

11. Fuchs B, Steinmann SP, Bishop AT. Free vascularized corticoperiosteal bone graft for the treatment of persistent nonunion of the clavicle. J Shoulder Elbow Surg. 2005; 14: 264-8

12. Havet E, Duparc F, Tobenas-Dujardin AC, Muller JM, Delas B, Fréger P. Vascular anatomical basis of clavicular non-union. Surg Radiol Anat. 2008; 30: 23-8.

13. Metsemakers WJ, Onsea J, Neutjens E, Steffens E, Schuermans A, McNally M, et al. Prevention of fracture-related infection: a multidisciplinary care package. Int Orthop. 2017; 41: 2457-69.

14. Bose D, Kugan R, Stubbs D, McNally M. Management of infected nonunion of the long bones by a multidisciplinary team. Bone Joint J. 2015; 97-B: 814-7. 